ANNALES

POLONICI MATHEMATICI

$81.3(2003)$

\title{
A generalization of the maximum principle to nonlinear parabolic systems
}

\author{
by Dmitry Portnyagin (Lviv)
}

\begin{abstract}
A generalization of the well-known weak maximum principle is established for a class of quasilinear strongly coupled parabolic systems with leading terms of $p$ Laplacian type.
\end{abstract}

1. Introduction. The maximum principle is one of the most important tools in the theory of partial differential equations (see the well-known book by Protter and Weinberger [10]). This principle is a generalization of the elementary fact of calculus that any function $f$ satisfying the inequality $f^{\prime \prime}>0$ on the interval $[a, b]$ achieves its maximum value at one of the endpoints of the interval. Many problems associated with differential equations of elliptic, parabolic and hyperbolic types exhibit maximum principles.

The maximum principle may be used to obtain results on approximation and uniqueness of solutions of differential equations.

Though the maximum principle for Laplace's equation has been known for many years, maximum principles for general second-order elliptic and parabolic operators were established quite recently.

Concerning systems of differential equations, until now the maximum principle has been extended only to a special class of systems of parabolic equations, the so-called weakly coupled systems. The system is said to be weakly coupled if it is coupled only through the terms which are not differentiated, each equation containing derivatives of just one component.

An extensive study of such systems was given in [7], where the authors maintain that their results cover all forms of maximum principle known by that time in the literature. They considered systems whose solutions satisfy a system of inequalities of the form

$$
L_{1}\left[u_{1}\right]+\sum_{i=1}^{n} h_{1 i} u_{i} \geq 0, \quad \ldots, \quad L_{n}\left[u_{n}\right]+\sum_{i=1}^{n} h_{n i} u_{i} \geq 0,
$$

2000 Mathematics Subject Classification: Primary 35B50, 35K40, 35K55.

Key words and phrases: parabolic systems, maximum principle. 
where $h_{i j}=h_{i j}(x, t)$ are functions of coordinates and time, and $L_{j}$ are conventional parabolic operators. Their technique is based upon analytic semigroup approach and the Tikhonov Fixed Point Theorem. As a most recent publication dealing with such systems we mention [4].

Consideration of these systems has been motivated by the fact that the maximum principle for them is a straightforward consequence and direct generalization of that for a single equation. The approaches to systems of this kind hinge upon attempting to formulate for such a system the maximum principle in exactly the same form known for a single scalar equation, i.e. for each component of a solution separately. In the present paper we take a different approach.

For strongly coupled or nondiagonal systems of differential equations, a counterexample given in [10] shows that the maximum principle no longer holds for a system in which the coupling is greater than in non-differentiated terms. Since the publication of De Giorgi's example of an unbounded solution to a linear elliptic system with bounded coefficients [2], it has become clear that extension of the maximum principle to strongly coupled systems is possible only for systems possessing a quite special structure. Nečas and Stará [8] were the first to obtain such results for a nonlinear nondiagonal elliptic system with coefficients depending on coordinates and unknowns, of the following structure:

$$
\sum_{i=1}^{n} \frac{\partial}{\partial x_{i}}\left(\sum_{j=1}^{n} \sum_{k=1}^{m} a_{i j}^{k r}(x, u) \frac{\partial}{\partial x_{j}} u_{k}\right)=\sum_{i=1}^{n} \frac{\partial}{\partial x_{i}} f_{i r}, \quad r=1, \ldots, m,
$$

under the following condition on the matrix of coefficients:

$$
\begin{gathered}
a_{i j}^{k r}(x, \xi)=\delta_{k r} A_{i j}^{k}(x, \xi)+b_{i j}^{k r}(x, \xi) \quad \text { for all } r=1, \ldots, m \text { and } \xi \in \mathbb{R}^{m} ; \\
\exists M>0 \quad b_{i j}^{k r}(x, \xi) \equiv 0 \quad \text { for }\left|\xi^{k}\right| \geq M .
\end{gathered}
$$

There exists yet another formulation of the maximum principle for a system. It concerns not each component separately, but the sum of the squares of the components of a solution. This maximum principle is valid for diagonal systems which on freezing the leading coefficients and discarding the right-hand sides and lower order terms reduce to just one single equation rewritten several times for all the unknown functions.

In the present paper we further investigate this matter. Namely, although restricting ourselves to systems of a quite special structure, we demonstrate in what way the maximum principle can be generalized to nonlinear parabolic systems of two equations in which coupling occurs in the leading derivatives.

To this end we employ the ideas set forth earlier in [5] and [9], and switch to new functions, for each of which the maximum principle is established 
in the classical form, whence we infer our final conclusion about the vector function solution itself. It should be stressed that for each component of the solution separately, the maximum principle in the classical formulation may not hold. To depict such a situation one can think of a system whose solutions are the functions sh and ch. The maximum principle states, roughly speaking, that a solution cannot be humpbacked, and does not hold for ch in the interval $(-1,1)$. Nevertheless, sh and ch could be decomposed into $\exp (x)$ and $\exp (-x)$, and the latter be regarded as new functions for each of which the principle would be valid in its usual form.

We also consider possible extensions to the case of three equations.

2. Basic considerations. In the present paper we shall be mainly concerned with systems of two equations, the model system being

$$
\left\{\begin{array}{l}
u_{t}=a_{1} \Delta_{p} u+b_{1} \Delta_{p} v+f_{1}, \\
v_{t}=a_{2} \Delta_{p} u+b_{2} \Delta_{p} v+f_{2}, \quad x \in Q
\end{array}\right.
$$

with the Dirichlet boundary conditions, understood in the weak sense:

$$
\left\{\begin{array}{l}
\left(u-g_{1}, v-g_{2}\right)(x, t) \in W_{0}^{1, p}(\Omega) \quad \text { a.e. } t \in(0, T), \\
(u, v)(x, 0)=(u, v)_{0}(x) .
\end{array}\right.
$$

Here $a_{i}$ and $b_{i}$ are some constants; $Q=(0, T] \times \Omega ; \partial Q \equiv\{\Omega \times\{0\}\} \cup$ $\{\partial \Omega \times(0, T]\} ; S=\partial \Omega \times(0, T] ; \Omega$ is a bounded domain in $\mathbb{R}^{n} ; T>0$; $x \in \Omega ; t \in(0, T] ; n>p \geq 2 ; \Delta_{p} u$ denotes the $p$-Laplacian of $u: \Delta_{p} u=$ $\operatorname{div}\left(|\nabla u|^{p-2} \nabla u\right) ; u, v \in C\left(0, T ; L^{2}(\Omega)\right) \cap L^{p}\left(0, T ; W^{1, p}(\Omega)\right) ; f_{1,2}=f_{1,2}(x) ;$ $W_{0}^{1, p}(\Omega)$ is the space of functions in $W^{1, p}(\Omega)$ with vanishing trace on $\partial \Omega$. For a positive number $q$ and a domain $Q$ we use the notation $\|\cdot\|_{q, Q}$ for the norm in the space $L^{q}(Q)$ and $\|\cdot\|_{\infty, Q}$ for the norm in $L^{\infty}(Q)$. Let us also define

$$
\begin{aligned}
& A_{i}^{(1)}=a_{1}|\nabla u|^{p-2} u_{x_{i}}+b_{1}|\nabla v|^{p-2} v_{x_{i}}, \\
& A_{i}^{(2)}=a_{2}|\nabla u|^{p-2} u_{x_{i}}+b_{2}|\nabla v|^{p-2} v_{x_{i}}, \quad i=1, \ldots, n .
\end{aligned}
$$

Throughout the paper the following assumptions are valid:

$$
\begin{gathered}
\min \left[\left(a_{1}-a_{2}\right)+\left(a_{2}-b_{1}\right) / p ;\left(b_{2}-b_{1}\right)+\left(b_{1}-a_{2}\right) / p\right] \geq 0 \\
f_{1,2} \in L^{\tau}(Q) \quad \text { for some } \tau>n / p+1 \\
g_{1,2} \in L^{\infty}(S), \quad(u, v)_{0} \in L^{\infty}(\Omega) .
\end{gathered}
$$

By parabolicity of system (2.1) we mean that the part without derivatives with respect to time is elliptic. The ellipticity is understood in the following sense, as introduced in [1]:

$$
\exists \lambda>0, \forall s_{j}^{i} \in \mathbb{R}^{2 n}, j=1,2, i=1, \ldots, n, \quad A_{i}^{(j)}(x, s) s_{j}^{i} \geq \lambda|s|^{p} .
$$

It is easy to check that under assumption $(*),(2.3)$ holds. We emphasize that we impose neither the Legendre nor the Legendre-Hadamard condition. 
A solution to system (2.1) with Dirichlet data (2.2) is understood in the weak sense, as in [3].

Definition 2.1. A measurable vector function $\left(u^{1}, u^{2}\right)=(u, v)$ is called a weak solution of problem (2.1)-(2.2) if

$$
u^{j} \in C\left(0, T ; L^{2}(\Omega)\right) \cap L^{p}\left(0, T ; W^{1, p}(\Omega)\right)
$$

and for all $t \in(0, T]$,

$$
\int_{\Omega} u^{j} \varphi_{j}(x, t) d x+\iint_{\Omega \times(0, t]}\left\{-u^{j} \varphi_{j t^{\prime}}+A_{i}^{(j)} \varphi_{j} x_{i}\right\} d x d t^{\prime}=\int_{\Omega} u_{0}^{j} \varphi_{j}(x, 0) d x
$$

for all test functions

$$
\varphi \in C\left(0, T ; L^{2}(\Omega)\right) \cap L^{p}\left(0, T ; W_{0}^{1, p}(\Omega)\right), \quad \varphi \geq 0 .
$$

The boundary condition in (2.2) is meant in the weak sense.

Definition 2.2. Let $u \in C\left(0, T ; L^{2}(\Omega)\right) \cap L^{p}\left(0, T ; W^{1, p}(\Omega)\right)$ and $\varphi_{+}=$ $\max [\varphi, 0]$. We define

$$
\begin{aligned}
& \sup _{\partial Q} u=\inf \left\{l \in \mathbb{R} \mid(u-l)_{+} \in W_{0}^{1, p}(\Omega) \text { for a.e. } t \in(0, T)\right. \text { and } \\
& \left.(u-l)_{+}=0 \text { a.e. in }\{0\} \times \Omega\right\} \text {; } \\
& \sup _{Q} u=\inf \left\{l \in \mathbb{R} \mid(u-l)_{+}=0 \text { a.e. in } Q\right\} ; \\
& \inf _{\partial Q} u=\sup \left\{l \in \mathbb{R} \mid(l-u)_{+} \in W_{0}^{1, p}(\Omega) \text { for a.e. } t \in(0, T)\right. \text { and } \\
& \left.(l-u)_{+}=0 \text { a.e. in }\{0\} \times \Omega\right\} \text {; } \\
& \inf _{Q} u=\sup \left\{l \in \mathbb{R} \mid(l-u)_{+}=0 \text { a.e. in } Q\right\} .
\end{aligned}
$$

Our present purpose is to extend the well-known weak maximum principle to the case of quasilinear strongly coupled parabolic systems (2.1). We develop the idea set forth in [5], [6], [9].

To begin with, consider a homogeneous system. Multiplying the first equation by $\alpha$ and adding the second one, we get

$$
\alpha u_{t}+v_{t}=\left(\alpha a_{1}+a_{2}\right) \Delta_{p} u+\left(\alpha b_{1}+b_{2}\right) \Delta_{p} v
$$

Let us consider $\alpha$ subject to the conditions:

$$
\left\{\begin{array}{l}
\alpha a_{1}+a_{2}=\gamma \alpha^{p-1} \\
\alpha b_{1}+b_{2}=\gamma, \quad \gamma>0
\end{array}\right.
$$

Here, as usual, it is understood that $\alpha^{p-1}=|\alpha|^{p-2} \alpha$. If there exists an $\alpha$ satisfying the nonlinear system (2.5) with some $\gamma>0$, then (2.4) takes the form

$$
\alpha u_{t}+v_{t}=\gamma \Delta_{p}(\alpha u)+\gamma \Delta_{p} v
$$

Now, suppose that at some points of $Q$ the linear combination $\alpha u+v$ is greater than $k$, its supremum on the parabolic boundary $\partial Q$. Multiplying 
both sides of $(2.6)$ by $\varphi=((\alpha u+v)-k)_{+}$and integrating with respect to $x$ over $\Omega$, and with respect to $t$ from 0 to $T$, we obtain

$$
\begin{aligned}
\int_{0}^{T} d t \int_{A(t)} d x\left(\alpha u_{t}+v_{t}\right)(\alpha u+v-k)=\frac{1}{2} \int_{A(T)} d x(\alpha u+v-k)^{2} \\
=-\gamma \int_{0}^{T} d t \int_{A(t)} d x\left\langle|\nabla \alpha u|^{p-2} \nabla \alpha u+|\nabla v|^{p-2} \nabla v, \nabla(\alpha u+v)\right\rangle,
\end{aligned}
$$

or

$$
\begin{aligned}
& \frac{1}{2} \int_{A(T)} d x(\alpha u+v-k)^{2} \\
& \quad+\gamma \int_{0}^{T} d t \int_{A(t)} d x\left\langle|\nabla \alpha u|^{p-2} \nabla \alpha u+|\nabla v|^{p-2} \nabla v, \nabla \alpha u+\nabla v\right\rangle=0,
\end{aligned}
$$

where $A(t) \equiv\{x \in \Omega \mid(\alpha u+v)(x, t) \geq k\} ;\langle\cdot, \cdot\rangle$ stands for the scalar product. We shall make use of the following

Lemma 2.3. Let $p \geq 2$. Then for any $\vec{a}, \vec{b} \in \mathbb{R}^{n}$,

$$
\left\langle|\vec{a}|^{p-2} \vec{a}+|\vec{b}|^{p-2} \vec{b}, \vec{a}+\vec{b}\right\rangle \geq \frac{1}{2^{p-1}}|\vec{a}+\vec{b}|^{p},
$$

where the constant $1 / 2^{p-1}$ is sharp.

A proof of the lemma is given in Section 10; see also [3, p. 13, Lemma 4.4].

Hence, it follows from (2.7) that

$$
\int_{0}^{T} d t \int_{A(t)} d x|\nabla(\alpha u+v)|^{p} \leq 0,
$$

and by the embedding theorem we get

$$
\begin{aligned}
C \int_{0}^{T} d t \int_{A(t)} d x|\alpha u+v-k|^{p} & \leq \int_{0}^{T} d t \int_{A(t)} d x|\nabla(\alpha u+v-k)|^{p} \\
& =\int_{0}^{T} d t \int_{A(t)} d x|\nabla(\alpha u+v)|^{p} \leq 0,
\end{aligned}
$$

where $C$ is a constant. Thus we conclude that

$$
\sup _{Q}(\alpha u+v) \leq \sup _{\partial Q}(\alpha u+v) \text {. }
$$

Similarly, testing $(2.6)$ on the function $\varphi=(k-(\alpha u+v))_{+}$we obtain

$$
\inf _{Q}(\alpha u+v) \geq \inf _{\partial Q}(\alpha u+v) \text {. }
$$


Analogously, by multiplying the first equation of (2.1) by $\alpha$ and subtracting the second, we arrive at

$$
\sup _{Q}(\alpha u-v) \leq \sup _{\partial Q}(\alpha u-v), \quad \inf _{Q}(\alpha u-v) \geq \inf _{\partial Q}(\alpha u-v),
$$

provided $\alpha$ satisfies the system

$$
\left\{\begin{array}{l}
\alpha a_{1}-a_{2}=\gamma \alpha^{p-1} \\
\alpha b_{1}-b_{2}=-\gamma, \quad \gamma>0
\end{array}\right.
$$

Summing up, we have

$$
\inf _{\partial Q}\left(\alpha_{1} u+v\right) \leq \alpha_{1} u+v \leq \sup _{\partial Q}\left(\alpha_{1} u+v\right) \quad \text { for a.e. }(x, t) \in Q
$$

with $\alpha_{1}$ from (2.3); and

$$
\inf _{\partial Q}\left(\alpha_{2} u-v\right) \leq \alpha_{2} u-v \leq \sup _{\partial Q}\left(\alpha_{2} u-v\right) \quad \text { for a.e. }(x, t) \in Q
$$

with $\alpha_{2}$ from (2.8). Hence, in particular, the estimates for $|u|$ and $|v|$ follow:

$$
\begin{aligned}
|u| \leq \frac{1}{\left|\alpha_{1}+\alpha_{2}\right|} \max \left\{\mid \sup _{\partial Q}\left(\alpha_{1} u+v\right)+\right. & \sup _{\partial Q}\left(\alpha_{2} u-v\right) \mid \\
& \left.\left|\inf _{\partial Q}\left(\alpha_{1} u+v\right)+\inf _{\partial Q}\left(\alpha_{2} u-v\right)\right|\right\},
\end{aligned}
$$

or

$$
\begin{aligned}
|u| \leq \frac{2}{\left|\alpha_{1}+\alpha_{2}\right|} \max \left\{\left|\sup _{\partial Q}\left(\alpha_{1} u+v\right)\right|, \mid\right. & \left|\sup _{\partial Q}\left(\alpha_{2} u-v\right)\right|, \\
& \left.\left|\inf _{\partial Q}\left(\alpha_{1} u+v\right)\right|,\left|\inf _{\partial Q}\left(\alpha_{2} u-v\right)\right|\right\} ;
\end{aligned}
$$

and

$$
\begin{aligned}
|v| \leq \frac{1}{\left|\alpha_{1}+\alpha_{2}\right|} \max \{\mid & \alpha_{2} \sup _{\partial Q}\left(\alpha_{1} u+v\right)-\alpha_{1} \inf _{\partial Q}\left(\alpha_{2} u-v\right) \mid, \\
& \left|\alpha_{2} \inf _{\partial Q}\left(\alpha_{1} u+v\right)-\alpha_{1} \sup _{\partial Q}\left(\alpha_{2} u-v\right)\right|, \\
& \left|\alpha_{2} \sup _{\partial Q}\left(\alpha_{1} u+v\right)-\alpha_{1} \sup _{\partial Q}\left(\alpha_{2} u-v\right)\right|, \\
& \left.\left|\alpha_{2} \inf _{\partial Q}\left(\alpha_{1} u+v\right)-\alpha_{1} \inf _{\partial Q}\left(\alpha_{2} u-v\right)\right|\right\},
\end{aligned}
$$

or

$$
\begin{array}{r}
|v| \leq \frac{2}{\left|\alpha_{1}+\alpha_{2}\right|} \max \left\{\left|\alpha_{2}\right|\left|\sup _{\partial Q}\left(\alpha_{1} u+v\right)\right|,\left|\alpha_{1}\right|\left|\inf _{\partial Q}\left(\alpha_{2} u-v\right)\right|\right. \\
\left.\left|\alpha_{2}\right| \inf _{\partial Q}\left(\alpha_{1} u+v\right)|,| \alpha_{1}|| \sup _{\partial Q}\left(\alpha_{2} u-v\right) \mid\right\}
\end{array}
$$

for a.e. $(x, t) \in Q$. 
3. The case $p=2$. For this case system (2.1) becomes linear and (2.5) takes the form

$$
\left\{\begin{array}{l}
\alpha a_{1}+a_{2}=\gamma \alpha \\
\alpha b_{1}+b_{2}=\gamma, \quad \gamma>0
\end{array}\right.
$$

and thus reduces to the quadratic equation

$$
\alpha^{2} b_{1}+\alpha\left(b_{2}-a_{1}\right)-a_{2}=0 .
$$

Its solutions are

$$
\begin{aligned}
& \alpha_{1(1,2)}=\frac{\left(a_{1}-b_{2}\right) \pm \sqrt{\left(a_{1}-b_{2}\right)^{2}+4 a_{2} b_{1}}}{2 b_{1}}, \\
& \gamma_{1(1,2)}=\frac{\left(a_{1}+b_{2}\right) \pm \sqrt{\left(a_{1}-b_{2}\right)^{2}+4 a_{2} b_{1}}}{2},
\end{aligned}
$$

with $a_{i}$ and $b_{i}$ satisfying the conditions

$$
\begin{gathered}
\left(a_{1}-b_{2}\right)^{2}>-4 a_{2} b_{1}, \\
\left(a_{1}+b_{2}\right)\left(a_{1} b_{2}-a_{2} b_{1}\right)>0 .
\end{gathered}
$$

Similarly, from (2.8) we get

$$
\begin{gathered}
\alpha_{2(1,2)}=\frac{\left(b_{2}-a_{1}\right) \pm \sqrt{\left(a_{1}-b_{2}\right)^{2}+4 a_{2} b_{1}}}{2 b_{1}}, \\
\gamma_{2(1,2)}=\frac{\left(a_{1}+b_{2}\right) \pm \sqrt{\left(a_{1}-b_{2}\right)^{2}+4 a_{2} b_{1}}}{2} .
\end{gathered}
$$

The condition $(* *)$ guarantees that $\gamma>0$, and $(*)$ that $\alpha_{1} \neq \alpha_{2}$.

ExAMPLE. Taking for instance

$$
a_{1}=5, \quad a_{2}=-4, \quad b_{1}=-1, \quad b_{2}=1,
$$

we have

$$
\begin{array}{ll}
\alpha_{1}=2(\sqrt{2}-1), & \gamma_{1}=3-2 \sqrt{2}>0, \\
\alpha_{2}=2(\sqrt{2}+1), & \gamma_{2}=3+2 \sqrt{2}>0,
\end{array}
$$

hence both (i) and (ii) are satisfied.

4. The case $p=2+\varepsilon, \varepsilon$ small enough. Let us consider slight deviations of $p$ from $p=2$. We have

$$
\left\{\begin{array}{l}
\alpha a_{1}+a_{2}=\gamma \alpha^{p-1} \\
\alpha b_{1}+b_{2}=\gamma
\end{array}\right.
$$

and hence

$$
\alpha a_{1}+a_{2}=\left(\alpha b_{1}+b_{2}\right) \alpha^{p-1} .
$$

Let us put $p=2+\varepsilon$. Then, taking the logarithms of both sides and representing

$$
\alpha=\alpha_{2}+\alpha_{\varepsilon}
$$


where $\alpha_{2}$ stands for the solution of (2.5) with $p=2$, we get

$$
\ln \left|\alpha_{2} a_{1}+a_{2}+\alpha_{\varepsilon} a_{1}\right|=\ln \left|\alpha_{2} b_{1}+b_{2}+\alpha_{\varepsilon} b_{1}\right|+(1+\varepsilon) \ln \left|\alpha_{2}+\alpha_{\varepsilon}\right| .
$$

Expanding in a series and equating the terms of the same order with respect to $\varepsilon$, we obtain

$$
\alpha_{\varepsilon}\left[\frac{a_{1}}{\left(\alpha_{2} a_{1}+a_{2}\right)}-\frac{b_{1}}{\left(\alpha_{2} b_{1}+b_{2}\right)}-\frac{1}{\alpha_{2}}\right]=\varepsilon \ln \alpha_{2} .
$$

Hence

$$
\alpha_{\varepsilon}=\frac{\left(\alpha_{2} a_{1}+a_{2}\right)\left(\alpha_{2} b_{1}+b_{2}\right) \alpha_{2} \ln \alpha_{2}}{\alpha_{2}\left[b_{2} b_{1}+a_{1} b_{2}-b_{1} a_{2}-a_{2}-b_{2}\right]-a_{2}\left(a_{1}+b_{2}\right)}(p-2) .
$$

Proceeding in much the same way, it is not difficult to find the next terms of the expansion.

5. Solutions of system (2.5). System (2.5) results in the equation $b_{1}|\alpha|^{p}+b_{2}|\alpha|^{p-2} \alpha-a_{1} \alpha-a_{2}=0$ under the condition $b_{1} \alpha+b_{2}>0$. Define

$$
\begin{aligned}
f(\alpha) & =b_{1}|\alpha|^{p}+b_{2}|\alpha|^{p-2} \alpha-a_{1} \alpha-a_{2}, \\
f^{\prime}(\alpha) & =p b_{1}|\alpha|^{p-2} \alpha+(p-1) b_{2}|\alpha|^{p-2}-a_{1}, \\
f^{\prime \prime}(\alpha) & =p(p-1) b_{1}|\alpha|^{p-2}+(p-1)(p-2) b_{2}|\alpha|^{p-4} \alpha \\
& =(p-1)|\alpha|^{p-4} \alpha\left\{p b_{1} \alpha+(p-2) b_{2}\right\} .
\end{aligned}
$$

From (5.3) it is easily seen that $f(\alpha)=0$ may have at most four roots.

To begin with, consider the (quite possible) situation when the zeros of both $f^{\prime}(\alpha)$ and $f(\alpha)$ coincide at some point. That is,

$$
\begin{array}{r}
b_{1}|\alpha|^{p}+b_{2}|\alpha|^{p-2} \alpha-a_{1} \alpha-a_{2}=0, \\
p b_{1}|\alpha|^{p-2} \alpha+(p-1) b_{2}|\alpha|^{p-2}-a_{1}=0,
\end{array}
$$

for some $\alpha$. Then from (5.2) we get

$$
|\alpha|^{p-2}=\frac{a_{1}}{p b_{1} \alpha+(p-1) b_{2}}
$$

and substituting into (5.1) we obtain

$$
(p-1) a_{1} b_{1} \alpha^{2}+\left(p a_{2} b_{1}+(p-2) a_{1} b_{2}\right) \alpha+(p-1) a_{2} b_{2}=0 .
$$

By solving this quadratic equation we get two roots:

$$
\begin{aligned}
& \alpha_{1,2}=-\frac{p}{2(p-1)} \frac{a_{2}}{a_{1}}-\frac{p-2}{2(p-1)} \frac{b_{2}}{b_{1}} \pm \sqrt{D}, \\
& D=\left[\frac{p}{2(p-1)} \frac{a_{2}}{a_{1}}+\frac{p-2}{2(p-1)} \frac{b_{2}}{b_{1}}\right]^{2}-\frac{a_{2} b_{2}}{a_{1} b_{1}} .
\end{aligned}
$$

Now it becomes obvious that the above-described situation occurs provided that either $\alpha_{1}$ or $\alpha_{2}$ (or both) solves the equation

$$
f^{\prime}(\alpha)=p b_{1}|\alpha|^{p-2} \alpha+(p-1) b_{2}|\alpha|^{p-2}-a_{1}=0 .
$$


This argument provides us with an idea how to proceed in order to determine conditions for $f(\alpha)$ to have at least two distinct zeros with

$$
\gamma=b_{1} \alpha+b_{2}>0 \text {. }
$$

Take for definiteness the case of $b_{1}<0$. Then $f( \pm \infty)$ is negative. If $f(\alpha)>0$ at the point where $f^{\prime}(\alpha)=0$, then we shall have at least two zeros of $f(\alpha)$. Substituting $|\alpha|^{p-2}$ from $f^{\prime}(\alpha)=0$ into $f(\alpha)>0$ we get

$$
\begin{aligned}
-\left\{(p-1) b_{1} \alpha^{2}+\left(p a_{2} b_{1} / a_{1}\right.\right. & \left.+(p-2) b_{2}\right) \alpha \\
+ & \left.(p-1) a_{2} b_{2} / a_{1}\right\} \frac{a_{1}}{p b_{1} \alpha+(p-1) b_{2}}>0,
\end{aligned}
$$

or, since $a_{1} /\left(p b_{1} \alpha+(p-1) b_{2}\right)$ is always positive for $\alpha \in \mathbb{R}$,

$$
(p-1) b_{1} \alpha^{2}+\left(p a_{2} b_{1} / a_{1}+(p-2) b_{2}\right) \alpha+(p-1) a_{2} b_{2} / a_{1}<0 .
$$

And (5.4) must have at least one solution in $\left(-\infty, \alpha_{1}\right] \cup\left[\alpha_{2}, \infty\right)$. If this is satisfied, then $f(\alpha)=0$ has at least two distinct roots. When we add the condition $\gamma=b_{1} \alpha+b_{2}>0$, we have to consider separately the cases $f\left(x_{0}\right)<0$ and $f\left(x_{0}\right)>0, x_{0}=-b_{2} / b_{1}$.

In the first case, (5.4) must have at least one solution in $A=\left(-\infty, x_{0}\right] \cap$ $\left\{\left(-\infty, \alpha_{1}\right] \cup\left[\alpha_{2}, \infty\right)\right\}$. For the second case we must additionally demand that $f^{\prime}(\widetilde{\alpha})=0$ for some $\widetilde{\alpha} \in\left(\alpha_{1}, \min \left[x_{0}, \alpha_{2}\right]\right)$.

Let us consider in turn all the possibilities, that is, $b_{1}<0, b_{1}>0, D<0$, $D \geq 0, f\left(x_{0}\right)<0$ and $f\left(x_{0}\right)>0$.

1. $b_{1}<0$ and $f\left(x_{0}\right)<0$. To determine the conditions for $f^{\prime}(\alpha)$ to have zeros in $A$ we resort to the mean-value theorem. Let $x_{1}, x_{2}, x_{3}$ be the roots of $f^{\prime}(\alpha)=0$. The roots may coincide. Note that $0=\left|\widetilde{x}_{1}\right|<\left|\widetilde{x}_{2}\right|<\left|x_{0}\right|$, where $\widetilde{x}_{1}, \widetilde{x}_{2}$ are the extremum points of $f^{\prime}(\alpha)$. Consider in turn all the possible mutual positions of $x_{1}, x_{2}, x_{3}, \alpha_{1}, \alpha_{2}$ and $x_{0}$ when at least one $x_{i}$ belongs to $\left(-\infty, \alpha_{1}\right] \cup\left[\alpha_{2}, \infty\right)$. Suppose for the beginning that $x_{0}>\alpha_{2}$. First, notice that $x_{2} \in\left(-\infty, \alpha_{1}\right] \cup\left[\alpha_{2}, \infty\right) \wedge x_{3}>x_{0}$ is ruled out, because it would imply that $f\left(x_{0}\right)<0, x_{0} \in\left(x_{1}, x_{3}\right)$ and at the same time $f\left(x_{2}\right)>0$, $x_{2}$ being a local minimum point. By the continuity of $f(\alpha)$ and $f^{\prime}(\alpha)$, this is a contradiction. Second, one can similarly see that $x_{2} \in\left(-\infty, \alpha_{1}\right] \cup\left[\alpha_{2}, \infty\right)$ and either $x_{1}$ or $x_{2}$ in $\left(\alpha_{1}, \alpha_{2}\right)$ is also impossible.

So we are left with the following choices:

(a) $x_{1}, x_{2}, x_{3} \in\left(-\infty, \alpha_{1}\right]$;

(b) $x_{1}, x_{2} \in\left(-\infty, \alpha_{1}\right]$ and $x_{3} \in\left[\alpha_{2}, x_{0}\right]$;

(c) $x_{1} \in\left(-\infty, \alpha_{1}\right]$ and $x_{2}, x_{3} \in\left(\alpha_{1}, \alpha_{2}\right)$;

(d) $x_{1} \in\left(-\infty, \alpha_{1}\right]$ and $x_{2}, x_{3} \in\left[\alpha_{2}, x_{0}\right]$;

(e) $x_{1} \in\left(-\infty, \alpha_{1}\right], x_{2} \in\left(\alpha_{1}, \alpha_{2}\right)$ and $x_{3} \in\left[\alpha_{2}, x_{0}\right]$;

(f) $x_{1}, x_{2} \in\left(\alpha_{1}, \alpha_{2}\right)$ and $x_{3} \in\left[\alpha_{2}, x_{0}\right]$. 
When $x_{0}<\alpha_{1}$ or $\alpha_{1}<x_{0}<\alpha_{2}$ we get just (a). Thus (a)-(f) exhaust all possibilities.

The sign of $f(\alpha)$ at infinity is determined by that of $b_{1}$. Basing on the mean-value theorem we can conclude that $f^{\prime}(\alpha)$ has in $A$ zeros with the required properties if

$$
b_{1} \cdot f^{\prime}\left(\min \left[\alpha_{1}, x_{0}\right]\right)<0 \vee f^{\prime}\left(\alpha_{2}\right) \cdot f^{\prime}\left(\max \left[\alpha_{2}, x_{0}\right]\right)<0 \quad \text { for } D \geq 0,
$$

and

$$
b_{1} \cdot f^{\prime}\left(x_{0}\right)<0 \quad \text { for } D<0 .
$$

These conditions cover the cases of $x_{0}<\alpha_{1}, \alpha_{1}<x_{0}<\alpha_{2}$, and $x_{0}>\alpha_{2}$.

Now, we take up the case

2. $b_{1}<0$ and $f\left(x_{0}\right) \geq 0$. In this case for $f(\alpha)$ to have two distinct zeros with $\gamma>0, f^{\prime}(\alpha)$ must have $x_{1} \in\left(-\infty, \alpha_{1}\right]$ and $x_{2} \in\left[\alpha_{1}, \alpha_{2}\right]$, i.e. a local minimum at $x_{2} \in\left(x_{1}, x_{0}\right)$ must be negative. Due to the mean-value theorem this amounts to the condition

$$
b_{1} \cdot f^{\prime}\left(\alpha_{1}\right)<0 \wedge f^{\prime}\left(\min \left[\alpha_{2}, x_{0}\right]\right) \cdot f^{\prime}\left(\min \left[\alpha_{1}, x_{0}\right]\right)<0 \quad \text { for } D \geq 0 .
$$

For $D<0$ there are no choices.

For the case of $b_{1}>0$ we argue in pretty much the same way with the only difference that we stipulate from the very beginning that $f(\alpha)<0$ at a point where $f^{\prime}(\alpha)=0$. This yields:

3. $b_{1}>0$ and $f\left(x_{0}\right)>0$. We have

$$
b_{1} \cdot f^{\prime}\left(\max \left[\alpha_{2}, x_{0}\right]\right)<0 \vee f^{\prime}\left(\alpha_{1}\right) \cdot f^{\prime}\left(\min \left[\alpha_{1}, x_{0}\right]\right)<0 \quad \text { for } D \geq 0,
$$

and

$$
b_{1} \cdot f^{\prime}\left(x_{0}\right)<0 \quad \text { for } D<0 .
$$

4. $b_{1}>0$ and $f\left(x_{0}\right) \leq 0$. We have

$$
b_{1} \cdot f^{\prime}\left(\alpha_{2}\right)<0 \wedge f^{\prime}\left(\max \left[\alpha_{1}, x_{0}\right]\right) \cdot f^{\prime}\left(\max \left[\alpha_{2}, x_{0}\right]\right)<0 \quad \text { for } D \geq 0 .
$$

No choices for $D<0$.

Moreover, analyzing the expression (5.4), which is always positive, together with (5.5), we infer that the cases with $b_{2}<0 \wedge a_{1}<0$ are ruled out.

All the above-said can be summarized in the following

Assumptions.

1. $f^{\prime}\left(\min \left[\alpha_{1}, x_{0}\right]\right)>0 \vee f^{\prime}\left(\alpha_{2}\right) \cdot f^{\prime}\left(\max \left[\alpha_{2}, x_{0}\right]\right)<0$ for $D \geq 0$; $b_{1}<0$ and $f\left(x_{0}\right)<0$;

2. $f^{\prime}\left(x_{0}\right)>0$ for $D<0 ; b_{1}<0$ and $f\left(x_{0}\right)<0$; 
3. $f^{\prime}\left(\alpha_{1}\right)>0 \wedge f^{\prime}\left(\min \left[\alpha_{2}, x_{0}\right]\right) \cdot f^{\prime}\left(\min \left[\alpha_{1}, x_{0}\right]\right)<0$ for $D \geq 0$; $b_{1}<0$ and $f\left(x_{0}\right) \geq 0$;

4. $f^{\prime}\left(\max \left[\alpha_{2}, x_{0}\right]\right)<0 \vee f^{\prime}\left(\alpha_{1}\right) \cdot f^{\prime}\left(\min \left[\alpha_{1}, x_{0}\right]\right)<0$ for $D \geq 0$; $b_{1}>0$ and $f\left(x_{0}\right)>0$;

5. $f^{\prime}\left(x_{0}\right)<0$ for $D<0 ; b_{1}>0$ and $f\left(x_{0}\right)>0$;

6. $b_{1} \cdot f^{\prime}\left(\alpha_{2}\right)<0 \wedge f^{\prime}\left(\max \left[\alpha_{1}, x_{0}\right]\right) \cdot f^{\prime}\left(\max \left[\alpha_{2}, x_{0}\right]\right)<0$ for $D \geq 0$; $b_{1}>0$ and $f\left(x_{0}\right) \leq 0$;

7. $f^{\prime}\left(\alpha_{1}\right)=f^{\prime}\left(\alpha_{2}\right)=0 ;\left\{x_{0}>\alpha_{2}, b_{1}<0\right\} \vee\left\{x_{0}<\alpha_{1}, b_{1}>0\right\}$.

Choice 7 is self-evident, stipulating that $f(\alpha)$ touches the $x$-axis at two extrema.

The situation with $f(\alpha)$ having at least two distinct zeros with $\gamma>0$ takes place whenever one of the above assumptions is fulfilled.

6. The main result. Finally, we come to the following

THEOREm 6.1. Let $(u, v)$ be a solution to system (2.1). If there exist two different numbers $\alpha_{1}, \alpha_{2}$ satisfying

$$
\begin{gathered}
\left\{\begin{array}{l}
\alpha_{1} a_{1}+a_{2}=\gamma_{1} \alpha_{1}^{p-1}, \\
\alpha_{1} b_{1}+b_{2}=\gamma_{1}, \quad \gamma_{1}>0,
\end{array}\right. \\
\left\{\begin{array}{l}
\alpha_{2} a_{1}-a_{2}=\gamma_{2} \alpha_{2}^{p-1}, \\
\alpha_{2} b_{1}-b_{2}=-\gamma_{2}, \quad \gamma_{2}>0,
\end{array}\right.
\end{gathered}
$$

i.e. one of Assumptions 1-7 is fulfilled, then

$$
\left\|\alpha_{1} u+v\right\|_{\infty, Q} \leq C_{1}, \quad\left\|\alpha_{2} u-v\right\|_{\infty, Q} \leq C_{2},
$$

where the constants $C_{1,2}$ depend only on $p, n, f_{1,2} ;\left\|g_{1,2}\right\|_{\infty, S},\left\|u_{0}, v_{0}\right\|_{\infty, \Omega}$; $a_{1,2}, b_{1,2}$; the domain $Q$ and the constants in the embedding theorems, and are independent of $u$ and $v$.

To prove the theorem we need the well-known Stampacchia lemma:

LEMMA 6.2. Let $\psi$ be a nonnegative nondecreasing function defined on $\left[k_{0}, \infty\right)$ which satisfies

$$
\psi(l) \leq \frac{C}{(l-k)^{\vartheta}}\{\psi(k)\}^{\delta} \quad \text { for } l>k \geq k_{0},
$$

with $\vartheta>0$ and $\delta>1$. Then

$$
\psi\left(k_{0}+d\right)=0,
$$

where $d=C^{1 / \vartheta}\left\{\psi\left(k_{0}\right)\right\}^{(\delta-1) / \vartheta} 2^{\delta /(\delta-1)}$.

For the proof see $[1$, Lemma 4.1, p. 8]. We also make use of the following lemma (see [3, Prop. 3.1, p. 7]): 
LEMMA 6.3. If $u \in L^{\infty}\left(0, T ; L^{2}(\Omega)\right) \cap L^{p}\left(0, T ; W_{0}^{1, p}(\Omega)\right)$ then

$$
\int_{0}^{T} \int_{\Omega} u^{q} \leq C\left(\int_{0}^{T} \int_{\Omega}|\nabla u|^{p}\right)\left(\underset{0<t<T}{\operatorname{esssup}} \int_{\Omega}|u|^{2}\right)^{p / n}
$$

with $q=p(n+2) / n$ and a constant $C$ depending only on $p$ and $n$.

Proof of Theorem 6.1. Choosing $w \equiv \operatorname{sign}\left(\alpha_{1} u+v\right)\left(\left|\alpha_{1} u+v\right|-k\right)_{+}$as a test function with

$$
k \geq k_{0}=\max \left[\left\|\alpha_{1} g_{1}+g_{2}\right\|_{L^{\infty}(S)},\left\|\alpha_{1} u_{0}+v_{0}\right\|_{L^{\infty}(\Omega)}\right],
$$

where $\alpha_{1}$ stands for the solution to system $\left(2.5^{*}\right)$, and literally repeating the argument of Section 2, namely, multiplying the first equation by $\alpha_{1}$ and adding to the second, then integrating in $t$ from 0 to $t, t \leq T$, and in $x$ over the domain $\Omega$, after applying Lemma 2.1 we obtain

$$
\int_{\Omega} w^{2}+\int_{0}^{t} \int_{\Omega}|\nabla w|^{p} \leq C \int_{0}^{t} \int_{\Omega}\left(f_{1} \alpha_{1}+f_{2}\right) w \leq C \int_{0}^{t} \int_{\Omega} f w,
$$

where $f=f_{1} \alpha_{1}+f_{2}$ and $C$ is a constant. Since $t \in(0, T]$ is arbitrary, by taking supremum we have

$$
\sup _{0<t<T} \int_{\Omega} w^{2}+\int_{0}^{T} \int_{\Omega}|\nabla w|^{p} \leq C \int_{0}^{T} \int_{\Omega} f w .
$$

Applying Lemma 6.3 we obtain

$$
\|w\|_{q, Q} \leq\left(\sup _{0<t<T} \int_{\Omega} w^{2}+\int_{0}^{T} \int_{\Omega}|\nabla w|^{p}\right)^{(p+n) /(q n)} .
$$

With the help of this relation, from (6.1) we get

$$
\|w\|_{q, Q}^{n q /(n+p)} \leq C \int_{0}^{T} \int_{\Omega} f w .
$$

Making use of the generalized Hölder inequality with exponents $q, n / p+1$ and $\tau$ on the right-hand side we obtain

$$
\|w\|_{q, Q}^{n q /(n+p)} \leq C\|w\|_{q, Q}\|f\|_{\tau, Q}\left(\int_{0}^{T} \int_{\Omega} \chi_{A(k, t)}\right)^{1-1 / q-1 / \tau},
$$

where $\chi_{A(k, t)}$ is the characteristic function of the set $A(k, t)=\{x \in \Omega \mid$ $|\alpha u+v|(x, t) \geq k\}$. Set

$$
\psi(k)=\int_{0}^{T} \operatorname{mes} A(k, t) d t
$$


where mes stands for Lebesgue measure. From the last relation it follows that

Estimating

$$
\|w\|_{q, Q}^{n q /(n+p)-1} \leq C\{\psi(k)\}^{1-1 / q-1 / \tau} .
$$

$$
(l-k)\{\psi(l)\}^{1 / q}=(l-k)\left(\int_{0}^{T} \int_{\Omega} \chi_{A(l, t)}\right)^{1 / q}<\left(\int_{0}^{T} \int_{\Omega} w^{q} \chi_{A(l, t)}\right)^{1 / q}<\|w\|_{q, Q}
$$

we arrive at

$$
(l-k)^{n q /(n+p)-1}\{\psi(l)\}^{n /(n+p)-1 / q} \leq C\{\psi(k)\}^{1-1 / q-1 / \tau},
$$

where $l>k \geq k_{0}$, or, succinctly,

$$
\psi(l) \leq \frac{C}{(l-k)^{q}}\{\psi(k)\}^{\delta}
$$

with

$$
\delta=\left(1-\frac{n}{p(n+2)}-\frac{1}{\tau}\right) /\left(\frac{n}{n+p}-\frac{n}{p(n+2)}\right) .
$$

Since we have assumed that

$$
f_{1,2} \in L^{\tau}(Q) \quad \text { for some } \tau>n / p+1,
$$

it is not difficult to check that

$$
1-\frac{n}{p(n+2)}-\frac{1}{\tau}>\frac{n}{n+p}-\frac{n}{p(n+2)}, \quad \text { and hence } \quad \delta>1 .
$$

By Stampacchia's result, from (6.2) we conclude that

$$
\psi\left(k_{0}+d\right)=0
$$

for some $d$ sufficiently large, but finite, depending only on $p, n, f_{1,2}, F_{1,2}$; $a_{i}, b_{i}$; the domain $Q$ and the constants in the embedding theorems, and independent of $u$ and $v$. Analogously we proceed for $\alpha_{2} u-v$, where $\alpha_{2}$ stands for the solution to system $\left(2.8^{*}\right)$.

It is not difficult to see from the previous considerations that the same estimates hold for the components $(u, v)$ of the solution themselves. In fact,

$$
\begin{aligned}
\|u\|_{\infty} & =\left\|\alpha_{1} u+\alpha_{2} u\right\|_{\infty} /\left|\alpha_{1}+\alpha_{2}\right| \\
& =\left\|\left(\alpha_{1} u+v\right)+\left(\alpha_{2} u-v\right)\right\|_{\infty} /\left|\alpha_{1}+\alpha_{2}\right| \leq\left(C_{1}+C_{2}\right) /\left|\alpha_{1}+\alpha_{2}\right|, \\
\|v\|_{\infty} & =\left\|v+\alpha_{1} u-\alpha_{1} u\right\|_{\infty} \leq\left\|\alpha_{1} u\right\|_{\infty}+\left\|\alpha_{1} u+v\right\|_{\infty},
\end{aligned}
$$

and hence the statement follows.

7. General structure. Let us now turn to a general system

$$
\left\{\begin{array}{l}
u_{t}=\frac{\partial}{\partial x_{i}}\left(A_{i}^{(1)}\left(u_{x}, v_{x}\right)\right)+f_{1}(x), \\
v_{t}=\frac{\partial}{\partial x_{i}}\left(A_{i}^{(2)}\left(u_{x}, v_{x}\right)\right)+f_{2}(x), \quad x \in Q,
\end{array}\right.
$$


with the Dirichlet boundary conditions (2.2). Here and in what follows, the summation convention over $i$ is assumed. $A_{i}^{(1),(2)}\left(u_{x}, v_{x}\right)$ are measurable $\mathbb{R}^{n} \times \mathbb{R}^{n} \rightarrow \mathbb{R}$ functions that satisfy the ellipticity condition (2.3) and are assumed to be subject to the following structure conditions:

$$
\begin{aligned}
\mid \alpha_{j} A_{i}^{(1)}\left(u_{x}, v_{x}\right)+A_{i}^{(2)} & \left(u_{x}, v_{x}\right)-\left(\alpha_{j} a_{1}+a_{2}\right)|\nabla u|^{p-2} u_{x_{i}} \\
& -\left(\alpha_{j} b_{1}+b_{2}\right)|\nabla v|^{p-2} v_{x_{i}} \mid \\
\leq & \varepsilon_{j}\left|\nabla\left(\alpha_{j} u+v\right)\right|^{p-1}+F_{j}(x), \quad \varepsilon_{j} \leq 2^{-p} \gamma_{j},
\end{aligned}
$$

for some $a_{1,2}, b_{1,2}$ and $F_{1,2} \in L^{\theta}(Q), \theta \geq(p+n) /(p-1)$; here $\alpha_{1}, \gamma_{1}$ and $\alpha_{2}, \gamma_{2}$ are solutions to systems $\left(2.5^{*}\right)$ and $\left(2.8^{*}\right)$ respectively.

THEOREM 7.1. Under assumptions (7.2) and (**), for every solution $(u, v) \in C\left(0, T ; L^{2}(\Omega)\right) \cap L^{p}\left(0, T ; W^{1, p}(\Omega)\right)$ of $(7.1)$,

$$
\|u, v\|_{\infty, Q} \leq C
$$

where the constant $C$ depends only on $p, n, \varepsilon_{1,2} ; f_{1,2}, F_{1,2} ;\left\|g_{1,2}\right\|_{\infty, S}$, $\left\|u_{0}, v_{0}\right\|_{\infty, \Omega} ; a_{1,2}, b_{1,2} ;$ the domain $Q$ and the constants in the embedding theorems, but is independent of $u$ and $v$.

The proof is analogous to that of the previous theorem.

8. The case of three equations. Unfortunately the technique set above cannot be fully generalized to systems of three quasilinear equations in $u, v, w$, with nonlinearities of $p$-Laplacian type. Namely, consider the model system

$$
\left\{\begin{array}{l}
u_{t}=a_{1} \Delta_{p} u+b_{1} \Delta_{p} v+c_{1} \Delta_{p} w \\
v_{t}=a_{2} \Delta_{p} u+b_{2} \Delta_{p} v+c_{2} \Delta_{p} w \\
w_{t}=a_{3} \Delta_{p} u+b_{3} \Delta_{p} v+c_{3} \Delta_{p} w
\end{array}\right.
$$

where $a_{i}, b_{i}, c_{i}$ are constants. Suppose we have managed to select suitable multipliers $\alpha$ and $\beta$ satisfying

$$
\left\{\begin{array}{l}
\alpha a_{1}+\beta a_{2}+a_{3}=\gamma \alpha^{p-1}, \\
\alpha b_{1}+\beta b_{2}+b_{3}=\gamma \beta^{p-1} \\
\alpha c_{1}+\beta c_{2}+c_{3}=\gamma, \quad \gamma>0 .
\end{array}\right.
$$

Proceeding as above, we would obtain

$$
\begin{aligned}
& \frac{1}{2} \int_{A(T)} d x(\alpha u+\beta v+w-k)^{2} \\
& +\gamma \int_{0}^{T} d t \int_{A(t)} d x\left\langle|\nabla \alpha u|^{p-2} \nabla \alpha u+|\nabla \beta v|^{p-2} \nabla \beta v+|\nabla w|^{p-2} \nabla w,\right. \\
& \nabla \alpha u+\nabla \beta v+\nabla w\rangle=0 .
\end{aligned}
$$


Now, consider the expression

$$
\left\langle|\vec{a}|^{p-2} \vec{a}+|\vec{b}|^{p-2} \vec{b}+|\vec{c}|^{p-2} \vec{c}, \vec{a}+\vec{b}+\vec{c}\right\rangle, \quad \vec{a}, \vec{b}, \vec{c} \in \mathbb{R}^{n} .
$$

Let $\vec{c} \equiv \vec{a}, \vec{b} \equiv-|s| \vec{a}$. Then

$$
\left\langle|\vec{a}|^{p-2} \vec{a}+|\vec{b}|^{p-2} \vec{b}+|\vec{c}|^{p-2} \vec{c}, \vec{a}+\vec{b}+\vec{c}\right\rangle=\left[2-|s|^{p-1}\right][2-|s|]|\vec{a}|^{p}<0,
$$

provided $|s|<2$ and $|s|^{p-1}>2$ for $p>2$ (obviously, it is always possible to choose such an $s$ ).

The expression is still negative if $\vec{c}=\vec{a}+\vec{\epsilon}_{1}, \vec{b}=-|s| \vec{a}+\vec{\epsilon}_{2}$ with $\vec{\epsilon}_{1}, \vec{\epsilon}_{2}$ small. At the same time, it is evident that $\left\langle|\vec{a}|^{p-2} \vec{a}+|\vec{b}|^{p-2} \vec{b}+|\vec{c}|^{p-2} \vec{c}\right.$, $\vec{a}+\vec{b}+\vec{c}\rangle$ is always positive for $\vec{a}, \vec{b}, \vec{c}$ orthogonal. Thus the expression in the corresponding integral in (16) is not well defined.

Nevertheless, consider a system of the form

$$
\left\{\begin{array}{l}
u_{t}=\frac{\partial}{\partial x_{i}}\left(A_{i}^{(1)}\left(u_{x}, v_{x}, w_{x}\right)\right)+f_{1}(x), \\
v_{t}=\frac{\partial}{\partial x_{i}}\left(A_{i}^{(2)}\left(u_{x}, v_{x}, w_{x}\right)\right)+f_{2}(x), \\
w_{t}=\frac{\partial}{\partial x_{i}}\left(A_{i}^{(3)}\left(u_{x}, v_{x}, w_{x}\right)\right)+f_{3}(x), \quad f \in L^{\tau}(Q),
\end{array}\right.
$$

where $A_{i}^{(j)}\left(u_{x}, v_{x}, w_{x}\right)$ are measurable $\mathbb{R}^{n} \times \mathbb{R}^{n} \times \mathbb{R}^{n} \rightarrow \mathbb{R}$ functions satisfying the ellipticity condition (2.3) with $j=1,2,3$ and the following structure conditions of a special kind:

$$
\begin{aligned}
& \mid \alpha A_{i}^{(1)}\left(u_{x}, v_{x}, w_{x}\right)+A_{i}^{(2)}\left(u_{x}, v_{x}, w_{x}\right)-\lambda_{1} \alpha^{p-1}|\nabla u|^{p-2} u_{x_{i}} \\
&-\left.\lambda_{1}|\nabla v|^{p-2} v_{x_{i}}\left|\leq \varepsilon_{1}\right| \nabla(\alpha u+v)\right|^{p-1}+F_{1}(x), \\
& \mid \beta A_{i}^{(2)}\left(u_{x}, v_{x}, w_{x}\right)+A_{i}^{(3)}\left(u_{x}, v_{x}, w_{x}\right)-\lambda_{2} \beta^{p-1}|\nabla v|^{p-2} v_{x_{i}} \\
&-\left.\lambda_{2}|\nabla w|^{p-2} w_{x_{i}}\left|\leq \varepsilon_{2}\right| \nabla(\beta v+w)\right|^{p-1}+F_{2}(x), \\
&\left.\left|\gamma A_{i}^{(3)}\left(u_{x}, v_{x}, w_{x}\right)+A_{i}^{(1)}\left(u_{x}, v_{x}, w_{x}\right)-\lambda_{3} \gamma^{p-1}\right| \nabla w\right|^{p-2} w_{x_{i}} \\
&-\left.\lambda_{3}|\nabla u|^{p-2} u_{x_{i}}\left|\leq \varepsilon_{3}\right| \nabla(\gamma w+u)\right|^{p-1}+F_{3}(x), \quad \varepsilon_{j} \leq 2^{-p} \lambda_{j},
\end{aligned}
$$

for some $\alpha, \beta, \gamma, \lambda_{1,2,3}>0$ and $F_{1,2,3} \in L^{\theta}(Q), \theta \geq(p+n) /(p-1)$. For example, assumptions $(8.2)_{1}-(8.2)_{3}$ are satisfied for the model system $(8.1)$ with $\alpha=-c_{2} / c_{1}, \beta=-a_{3} / a_{2}, \gamma=-b_{1} / b_{3}$ provided that

$$
\begin{aligned}
& -\frac{c_{2}}{c_{1}}\left|\frac{c_{2}}{c_{1}}\right|^{p-2}=\frac{a_{2} c_{1}-c_{2} a_{1}}{b_{2} c_{1}-c_{2} b_{1}}, \quad\left(b_{2} c_{1}-c_{2} b_{1}\right) c_{1}>0, \\
& -\frac{a_{3}}{a_{2}}\left|\frac{a_{3}}{a_{2}}\right|^{p-2}=\frac{a_{2} b_{3}-b_{2} a_{3}}{a_{2} c_{3}-c_{2} a_{3}}, \quad\left(a_{2} c_{3}-c_{2} a_{3}\right) a_{2}>0,
\end{aligned}
$$




$$
\begin{aligned}
-\frac{b_{1}}{b_{3}}\left|\frac{b_{1}}{b_{3}}\right|^{p-2} & =\frac{b_{3} c_{1}-c_{3} b_{1}}{b_{3} a_{1}-a_{3} b_{1}}, \quad\left(b_{3} a_{1}-a_{3} b_{1}\right) b_{3}>0 \\
c_{2}, a_{3}, b_{1} & \neq 0 .
\end{aligned}
$$

By much the same arguments as above we can show that for every solution $(u, v, w) \in C\left(0, T ; L^{2}(\Omega)\right) \cap L^{p}\left(0, T ; W^{1, p}(\Omega)\right)$ of $(8.1)$ with Dirichlet data,

$$
\|\alpha u+v\|_{\infty} \leq C_{1}, \quad\|\beta u+v\|_{\infty} \leq C_{2}, \quad\|\gamma u+v\|_{\infty} \leq C_{3} .
$$

And hence it is not difficult to deduce that

$$
\|u, v, w\|_{\infty, Q} \leq C,
$$

where $C$ is a constant depending only on $p, n, \varepsilon_{1,2,3} ; f_{1,2,3}, F_{1,2,3} ; a_{1,2,3}$, $b_{1,2,3}, c_{1,2,3}$; the preassigned values of $u, v, w$ on $\partial Q$; the domain $Q$ and the constants in the embedding theorems, and are independent of $u, v$ and $w$.

There is yet another possibility of generalization to the case of three equations. Let us consider a system of the following structure:

$$
\left\{\begin{array}{l}
u_{t}=\frac{\partial}{\partial x_{i}}\left(F \cdot\left(a_{1} u_{x_{i}}+b_{1} v_{x_{i}}+c_{1} w_{x_{i}}\right)\right), \\
v_{t}=\frac{\partial}{\partial x_{i}}\left(F \cdot\left(a_{2} u_{x_{i}}+b_{2} v_{x_{i}}+c_{2} w_{x_{i}}\right)\right), \\
w_{t}=\frac{\partial}{\partial x_{i}}\left(F \cdot\left(a_{3} u_{x_{i}}+b_{3} v_{x_{i}}+c_{3} w_{x_{i}}\right)\right),
\end{array}\right.
$$

where $F=F\left(t, x, u, v, w, u_{x}, v_{x}, w_{x}\right)$ is a measurable bounded function $\mathbb{R} \times \mathbb{R}^{n} \times \mathbb{R}^{3} \times \mathbb{R}^{3 n} \rightarrow \mathbb{R}, F>0 ; a_{j}, b_{j}, c_{j}$ are constants, $j=1,2,3$.

Suppose that $\alpha$ and $\beta$ satisfy

$$
\left\{\begin{array}{l}
\alpha a_{1}+\beta a_{2}+a_{3}=\gamma \alpha \\
\alpha b_{1}+\beta b_{2}+b_{3}=\gamma \beta, \\
\alpha c_{1}+\beta c_{2}+c_{3}=\gamma, \quad \gamma>0
\end{array}\right.
$$

Hence we conclude that the solutions of (8.3) satisfy

$$
\begin{aligned}
& \inf _{\partial Q}\left(\alpha_{1} u+\beta_{1} v+w\right) \leq \alpha_{1} u+\beta_{1} v+w \leq \sup _{\partial Q}\left(\alpha_{1} u+\beta_{1} v+w\right), \\
& \inf _{\partial Q}\left(\alpha_{2} u+\beta_{2} v+w\right) \leq \alpha_{2} u+\beta_{2} v+w \leq \sup _{\partial Q}\left(\alpha_{2} u+\beta_{2} v+w\right), \\
& \inf _{\partial Q}\left(\alpha_{3} u+\beta_{3} v+w\right) \leq \alpha_{3} u+\beta_{3} v+w \leq \sup _{\partial Q}\left(\alpha_{3} u+\beta_{3} v+w\right),
\end{aligned}
$$

for a.e. $(x, t) \in Q$, provided that $\operatorname{det}\left(a_{j}, b_{j}, c_{j}\right) \neq 0$; here $\alpha_{1,2,3}$ and $\beta_{1,2,3}$ are three different solutions of system (8.4), i.e. the components of three linearly independent eigenvectors of the matrix $\left(a_{j}, b_{j}, c_{j}\right)$ corresponding to positive eigenvalues. It clearly follows that

$$
\|u\|_{\infty},\|v\|_{\infty},\|w\|_{\infty} \leq C
$$


with $C$ a constant depending on $a_{j}, b_{j}, c_{j}$ and the values of $u, v, w$ on $\partial Q$. We shall not be concerned with nonhomogeneous systems (8.3) and systems of more general structure.

9. Discussion. The main idea of our approach is as follows: instead of trying to establish the maximum principle for each component $u^{j}$ of the solution, we introduce some linear combinations of the components, in general some functions $H(t, x, u)$ of $t, x$ and $u^{j}$, for each of which the maximum principle holds and from whose boundedness we are able to derive estimates for the components of the solution, in much the same way as the maximum principle for a single equation enables us to do.

Let us consider a few examples. For simplicity we restrict ourselves to the case of one spatial variable and $(u, v) \in C^{0}(\partial Q \cup Q) \cap C^{2}(Q)$.

EXAMPLE 1.

$$
\left\{\begin{array}{l}
u_{t} \leq 7 u_{x x}-3 v_{x x}, \\
v_{t}=2 u_{x x}+2 v_{x x}, \quad(x, t) \in Q=\{1 \leq x \leq 2,0 \leq t \leq 1\} .
\end{array}\right.
$$

The substitution

$$
H_{1}=u-v, \quad H_{2}=2 u-3 v
$$

reduces the system to the form

$$
\left\{\begin{array}{l}
H_{1 t} \leq 5 H_{1 x x} \\
H_{2 t} \leq 4 H_{2 x x}
\end{array}\right.
$$

for which the maximum principle is obviously valid in the conventional form and which is satisfied by the functions

$$
H_{1}=\frac{1}{\sqrt{t}} \exp \left(-x^{2} / 20 t\right), \quad H_{2}=\frac{1}{\sqrt{t}} \exp \left(-x^{2} / 16 t\right) .
$$

EXAMPLE 2.

$$
\left\{\begin{array}{c}
u_{t} \leq-13 u_{x x}-20(t / x) v_{x x}-26(1 / x) u_{x} \\
v_{t} \leq 12(x / t) u_{x x}+18 v_{x x}+24(1 / t) u_{x}+(1 / t) v \\
(x, t) \in Q=\{1 \leq x \leq 2,1 \leq t \leq 2\}
\end{array}\right.
$$

The substitution

$$
H_{1}=3 x u+4 t v, \quad H_{2}=4 x u+5 t v
$$

reduces the system to the form

$$
\left\{\begin{array}{l}
H_{1 t} \leq 3 H_{1 x x}, \\
H_{2 t} \leq 2 H_{2 x x},
\end{array}\right.
$$

for which the maximum principle holds. 
EXAMPLE 3.

$$
\left\{\begin{array}{c}
u_{t} \leq 3 x(3-2 x t) u_{x x}+18(1-4 x) u_{x}+9 v-12 t u \\
v_{t} \leq 3 x(3-2 x t) v_{x x}+4 x^{2}(3-48 x+4 x t) u_{x}-12 x u+6 x^{2} v \\
(x, t) \in Q=\{1 / 2 \leq x \leq 1,1 / 2 \leq t \leq 1\}
\end{array}\right.
$$

By the substitution

$$
H_{1}=x u-t v, \quad H_{2}=2 x^{2} u-3 v
$$

this system takes the form

$$
\left\{\begin{array}{l}
H_{1 t} \leq 3 x(3-2 x t) H_{1 x x} \\
H_{2 t} \leq 3 x(3-2 x t) H_{2 x x}
\end{array}\right.
$$

for which the maximum principle is valid.

EXAMPLE 4.

$$
\left\{\begin{array}{c}
u_{t} \leq-x^{2} u_{x x}-2 v_{x x}-6 x u_{x}-(4 / x) v_{x}-6 u \\
v_{t} \leq 24 x^{4} u_{x x}+13 x^{2} v_{x x}+144 x^{3} u_{x}+26 x v_{x}+144 x^{2} u \\
(x, t) \in Q=\{1 \leq x \leq 2,0 \leq t \leq 1\}
\end{array}\right.
$$

The substitution

$$
H_{1}=4 x^{2} u+v, \quad H_{2}=3 x^{2} u+v
$$

reduces this system to the following form:

$$
\left\{\begin{array}{l}
H_{1 t} \leq \frac{\partial}{\partial x}\left(5 x^{2} H_{1 x}\right) \\
H_{2 t} \leq \frac{\partial}{\partial x}\left(7 x^{2} H_{2 x}\right)
\end{array}\right.
$$

Though this system is degenerate in $Q$, the maximum principle nevertheless holds.

EXAMPle 5. As the last example take the system from [10, p. 192]:

$$
\left\{\begin{array}{l}
u_{1 t} \leq u_{1 x x} \\
u_{2 t}=u_{2 x x}-9 u_{1 x}
\end{array}\right.
$$

or

$$
\left\{\begin{array}{l}
u_{1 t}=u_{1 x x}, \\
u_{2 t} \leq u_{2 x x}-9 u_{1 x}, \quad Q=\{0 \leq x \leq 1,0 \leq t \leq 1\}
\end{array}\right.
$$

the only difference with [10] being that we demand strict equality in one of the equations. The maximum principle in the above-stated form holds with

$$
H_{1}=u_{1}, \quad H_{2}=(9 / 2) x u_{1}-u_{2}
$$

for the first system, and with

$$
H_{1}=u_{1}, \quad H_{2}=u_{2}-(9 / 2) x u_{1}
$$

for the second one. In [10] this example is drawn to illustrate the violation of the maximum principle if the coupling is stronger than that in nondifferentiated terms. One can easily see that nevertheless the maximum principle 
in our form holds in $Q$ for the latter system, with the choice of functions as in $[10]$ :

$$
\begin{gathered}
u_{1}=-\exp (x+t), \quad u_{2}=t-4(x-1 / 2)^{2}, \\
H_{1}=u_{1}, \quad H_{2}=u_{2}-(9 / 2) x u_{1}=t-4(x-1 / 2)^{2}+(9 / 2) x \exp (x+t) .
\end{gathered}
$$

10. Proof of Lemma 2.3. We have

$$
\begin{aligned}
\left\langle|\vec{a}|^{p-2} \vec{a}+|\vec{b}|^{p-2} \vec{b}, \vec{a}+\vec{b}\right\rangle= & |\vec{a}|^{p}+|\vec{b}|^{p}+\left[|\vec{a}|^{p-2}+|\vec{b}|^{p-2}\right]\langle\vec{a}, \vec{b}\rangle \\
= & \frac{1}{2}\left[|\vec{a}|^{p-2}+|\vec{b}|^{p-2}\right](\vec{a}+\vec{b})^{2} \\
& +\frac{1}{2}\left[|\vec{b}|^{p}-|\vec{a}|^{p-2} \cdot|\vec{b}|^{2}+|\vec{a}|^{p}-|\vec{b}|^{p-2} \cdot|\vec{a}|^{2}\right] \\
= & \frac{1}{2}\left[|\vec{a}|^{p-2}+|\vec{b}|^{p-2}\right](\vec{a}+\vec{b})^{2} \\
& +\frac{1}{2}\left(|\vec{a}|^{p-2}-|\vec{b}|^{p-2}\right)\left(|\vec{a}|^{2}-|\vec{b}|^{2}\right) .
\end{aligned}
$$

The last expression is always greater than 0 for $p \geq 2$. Due to the inequality

$$
|\vec{a}|^{p-2}+|\vec{b}|^{p-2} \geq \frac{1}{2^{p-2}}|\vec{a}+\vec{b}|^{p-2}
$$

we thus get

$$
\left\langle|\vec{a}|^{p-2} \vec{a}+|\vec{b}|^{p-2} \vec{b}, \vec{a}+\vec{b}\right\rangle \geq \frac{1}{2^{p-1}}|\vec{a}+\vec{b}|^{p} .
$$

The present proof is simpler than that given in [3].

Acknowledgements. The author is greatly indebted to Professors P. Biler and W. Zajaczkowski for reading the manuscript and for their help during the preparation of this paper.

\section{References}

[1] Y. Z. Chen and L. C. Wu, Second Order Elliptic Equations and Elliptic Systems, Transl. Math. Monographs 174, Amer. Math. Soc. Providence, RI, 1998.

[2] E. De Giorgi, Un esempio di estremali discontinue per un problema variazionale di tipo ellittico, Boll. Un. Mat. Ital. 1 (1968), 135-137.

[3] E. DiBenedetto, Degenerate Parabolic Equations, Springer, New York, 1993.

[4] F. Dickstein and M. Escobedo, A maximum principle for semilinear parabolic systems and applications, Nonlinear Anal. 45 (2001), 825-837.

[5] L. Dung, Hölder regularity for certain strongly coupled parabolic systems, J. Differential Equations 151 (1999), 313-344.

[6] H. Hoshino, Nonnegative global solutions to a class of strongly coupled reactiondiffusion systems, Adv. Differential Equations 5 (2000), 801-832.

[7] G. Lu and B. D. Sleeman, Maximum principles and comparison theorems for semilinear parabolic systems and their applications, Proc. Roy. Soc. Edinburgh Sect. A 123 (1993), 857-885.

[8] J. Nečas and J. Stará, Principio di massimo per $i$ sistemi ellittici quasi-lineari non diagonali, Boll. Un. Mat. Ital. (4) 6 (1972), 1-10. 
[9] M. A. Pozio and A. Tesei, Global existence of solutions for a strongly coupled quasilinear parabolic system, Nonlinear Anal. 12 (1990), 657-689.

[10] M. H. Protter and H. F. Weinberger, Maximum Principles in Differential Equations, Prentice-Hall, Englewood Cliffs, NJ, 1967.

Institute for Physics of Condensed Matter

National Academy of Sciences of Ukraine

1 Svientsitskii St., 79011 Lviv, Ukraine

E-mail: mitport@hotmail.com

Reçu par la Rédaction le 15.4.2002

Révisé le 24.3.2003 